Sonderdruck aus

Gregor Feindt / Félix Krawatzek /

Daniela Mehler / Friedemann Pestel /

Rieke Trimçev (Hg.)

\title{
Europäische Erinnerung als verflochtene Erinnerung
}

Vielstimmige und vielschichtige

Vergangenheitsdeutungen jenseits der Nation

V\& R unipress

ISBN 978-3-8471-0052-2

ISBN 978-3-8470-0052-5 (E-Book) 



\section{Inhalt}

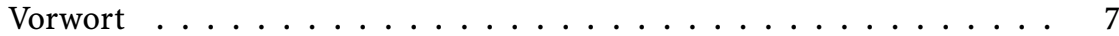

\section{Einleitung}

Gregor Feindt, Félix Krawatzek, Daniela Mehler, Friedemann Pestel, Rieke Trimçev

Europäische Erinnerung? Erinnerungsforschung jenseits der Nation . . . 11

\section{Erinnerung als Traditionsstiftung}

Klaus Oschema

Ein Karl für alle Fälle - Historiografische Verortungen Karls des Großen zwischen Nation, Europa und der Welt . . . . . . . . . . . . 39

Roland Scheel

Wikinger und Wikingerzeit - Der vormittelalterliche Norden als

Gegenstand europäischer Erinnerung? . . . . . . . . . . . . . 65

Simon Hadler

Zugehörigkeit durch Abgrenzung - Der Türke als der Andere Europas 93

\section{Erinnerung als Deutungsregie}

Friedemann Pestel

Versailles als memory building - Memory-building mit Versailles . . . . 121

\section{Erinnerung als Deutungskampf}

\section{Gregor Feindt}

Flucht und Vertreibung zwischen Kaltem Krieg und Universalisierung . . 153

Marcin Napiórkowski

Der Warschauer Aufstand im Ringen um europäische Identität . . . . . 179 
Daniela Mehler

Srebrenica und das Problem der einen Wahrheit . . . . . . . . . . . 205

\section{Schlussbetrachtung}

Gregor Feindt, Félix Krawatzek, Daniela Mehler, Friedemann Pestel, Rieke Trimçev

Funktionen europäischer Erinnerung in der postnationalen Konstellation 237

Oliver Dimbath

Nachwort aus sozialtheoretischer Perspektive . . . . . . . . . 265

Autorenverzeichnis . . . . . . . . . . . . . . . 273 
Schlussbetrachtung 



\section{Funktionen europäischer Erinnerung in der postnationalen Konstellation?}

In der Einleitung dieses Buches haben wir die Prämisse formuliert, dass europäische Erinnerung als Gegenstand kontroverser Auffassungen Teil der diskursiven Möglichkeiten unserer Zeit geworden ist. Jenseits der Frage nach Ideal oder Wirklichkeit haben die sieben Fallstudien europäische Erinnerung als semantische Realität verstanden, die es in ihrer Mehrdeutigkeit und Geschichtlichkeit zu erschließen galt. Die in der Einleitung entwickelten Heuristiken der Vielstimmigkeit und Vielschichtigkeit dienten dabei als Leitfaden für die Fallstudien und eröffnen nun abschließend eine vergleichende Perspektive.

Tatsächlich entwickeln sich im Vergleich einzelne Aspekte einer Fallstudie im Dialog mit einem oder mehreren anderen Beiträgen zu tertia comparationis, die den Gedankengang dieses Schlusskapitels strukturieren. Die folgenden Seiten fragen nach unterschiedlichen Mustern solcher Deutungen, die ihren Gegenstand gleichzeitig als vergangen und als europäisch repräsentieren. Erst innerhalb eines so aufgefächerten Blicks wird deutlich, um welche Kategorien, $\mathrm{Zu}$ schreibungen und letztlich Ansprüche heute gerungen wird, wenn von europäischer Erinnerung die Rede ist. Damit soll eine Charakterisierung von europäischer Erinnerung in ihrer postnationalen Konstellation vorgeschlagen werden.

Durch die unterschiedlichen Fallstudien hindurch sind regelmäßige Deutungsmuster europäischer Erinnerung zu erkennen, die sich in vier Kategorien unterteilen lassen. Erstens werden in ihnen die imaginierten Räume Europas verhandelt. Dies manifestiert sich anhand symbolischer und geografischer Grenzziehungen. Zweitens funktioniert Europa vielfältig als Kapital in Erinnerungsdiskursen. Drittens kann der Begriff Europa Erinnerung in politischideologischen Auseinandersetzungen mobilisieren. Viertens wird in den Deu-

1 Die folgenden Seiten formulieren Gedanken, die im Dezember 2012 im Rahmen eines Redaktionsworkshops am Zentrum für interdisziplinäre Forschung in Bielefeld aus der Diskussion mit allen Autorinnen und Autoren dieses Bandes sowie Oliver Dimbath, Helmut König und Harald Wydra entstanden sind. 
tungen der Vergangenheit Europas erprobt, wie heute mit seiner Heterogenität umgegangen werden kann. Europa steht hier für eine Veränderung im Erinnerungsdiskurs, die Heterogenität zum bewahrenswerten und eingehegten status quo von Erinnerung erhebt.

\section{Imaginierte Räume Europas}

Erinnerungen stellen Vergangenheit stets in räumlichen Kategorien vor. ${ }^{2}$ Eine solche "topologische Qualität «", um einen treffenden Ausdruck von Aleida Assmann aufzugreifen, zeigen auch die zeitgenössischen Erinnerungen, die als

2 Der Konnex von Erinnerungsprozessen und Raumvorstellungen hat historisch unterschiedliche Formen angenommen. Seit der Antike repräsentierte die ars memoria komplexe Bewusstseinsinhalte, indem sie deren Zusammenhang in Analogie zu bestimmten räumlichen Relationen in einer Landschaft oder einem Gebäude setzte, s. die exemplarische Studie von Stefan Goldmann, Topoi des Gedenkens. Pausanias' Reise durch die griechische Gedächtnislandschaft, in: Anselm Haverkamp/Renate Lachmann (Hrsg.), Gedächtniskunst. Raum Bild-Schrift, Frankfurt am Main 1991, S. 145 - 164, sowie maßgeblich Frances A. Yates, The Art of Memory, London 1966. Anders als diese intendierte Lokalisierung der Mnemotechnik stellen aber auch typische Metaphern wie Bibliothek, Labyrinth, Tempel oder Stadt vergangene Wirklichkeiten oder gar Erinnerungsprozesse selbst in räumlichen Figuren vor. Wie zum Beispiel Aleida Assmann oder Douwe Draaisma zeigen, suggerieren unterschiedliche Raummetaphern dabei implizit immer auch unterschiedliche Verständnisse der Formen und Funktionen von Erinnerungsprozessen. Aleida Assmann, Erinnerungsräume. Formen und Wandlungen des kulturellen Gedächtnisses, München 2003, S. 158-162, und Douwe Draaisma, Die Metaphernmaschine. Eine Geschichte des Gedächtnisses, Darmstadt 1999, S. 36 - 40 und S. 78-84. Auf der theoretischen Metaebene wird der Zusammenhang von Raum und Erinnerung dann besonders bei Maurice Halbwachs als Materialisierung ausformuliert, s. Stephan Egger, Auf den Spuren der "verlorenen Zeit«. Maurice Halbwachs und die Wege des »kollektiven Gedächtnisses«, in: Maurice Halbwachs, Stätten der Verkündigung im Heiligen Land. Eine Studie zum kollektiven Gedächtnis, hrsg. v. dems., Konstanz 2003, S. 219-268. Zwischen diesem soziologischen Zugriff, der Erinnerungssteuerung der lokalisierenden Mnemotechnik und der Naturalisierung räumlicher Erinnerungs- und Gedächtnismetaphern changieren räumliche Kategorien im Forschungsparadigma der lieux de mémoire, die nicht streng dem räumlichen locus der klassischen Mnemotechnik, sondern oft den imagines, also verorteten Begriffen und Vorstellungen, nachgehen, s. Martin Reisigl, Das Konzept der »lieux de mémoire " aus rhetorischer und linguistischer Perspektive, in: Benoît Majerus/Sonja Kmec/Michel Margue/Pit Péporté (Hrsg.), Dépasser le cadre national des "Lieux de mémoire«. Innovations méthodologiques, approches comparatives, lectures transnationales, Brüssel 2009, S. 117 - 140, hier S. 132, und Tilmann Robbe, Historische Forschung und Geschichtsvermittlung. Erinnerungsorte in der deutschsprachigen Geschichtswissenschaft, Göttingen 2009, S. 99-105. Zur "suggestiven Kraft dieser Unschärfen« s. auch Assmann, Erinnerungsräume, S. 158 - 162 und S. 298 -339. Besonders im Verhältnis zur Mnemotechnik dreht unser Argument an dieser Stelle freilich den funktionalen Zusammenhang um: Betrachtet wird nicht, wie Raumvorstellungen komplexe Erinnerungsleistungen unterstützen können. Gefragt wird vielmehr, wie bestimmte Erinnerungsmuster Raumvorstellungen bedingen und transportieren.

3 Assmann, Erinnerungsräume, S. 158. 
europäisch dargestellt werden. Sie lassen auf unterschiedlichen Ebenen Raumvorstellungen erkennen. Damit sind nicht allein im strengen Wortsinne geografische Vorstellungen der Grenzen und Binnenstruktur Europas gemeint. Vielmehr entstehen Verräumlichungen auf abstraktere Weise auch dort, wo Erinnerungen soziale Gruppen durch innere und äußere Raumbezüge in eine relationale Ordnung zu anderen sozialen Gruppen stellen. Die Europareferenz erhält dadurch jeweils Kontur und Dichte.

Die vorliegenden Fallstudien können hinsichtlich dreier wiederkehrender Erinnerungsmuster verglichen werden, für die räumliches Vorstellungsvermögen konstitutiv ist: Erstens finden sich unterschiedliche Figuren des Anderen, die für die Grenze dessen grundlegend sind, was als Europa vorgestellt werden kann. Zweitens geben innere Trennlinien Europa ein Zentrum und grenzen davon Peripherieregionen ab. Drittens beobachten die Fallstudien die expansive Tendenz eines solchen Zentrums.

Durch die Konstruktion von Alterität stellt eine erinnernde Gruppe bekanntlich Identität und Kohäsion her. ${ }^{4}$ Simon Hadlers Fallstudie zum Türken illustriert, wie durch ein als europäisch konnotiertes Deutungsmuster eine kontinuierliche Abgrenzung von einem Anderen vorgenommen wird, der außerhalb des als eigen verstandenen Raums verortet wird. Die so gezogene Trennlinie wird insbesondere dadurch wirkungsvoll, dass sie im Sinne des in der Einleitung als Traditionsstiftung bezeichneten Erinnerungsmodus eine Vielzahl an unterschiedlichen Projektionsmöglichkeiten zulässt und die Figur des Anderen verschiedenartig variiert. So ist der Türke eine historisch wiederholt verfügbare Chiffre, um jeweils gegenwärtige Feinde zu benennen, sie negativ zu besetzen und sich von ihnen abzugrenzen. Die in der Fallstudie ausgeführte Gleichsetzung des islamischen Fundamentalismus oder des imperialistischen Russlands - jeweils verstanden als Metapher für die Bedrohung Europas - mit den heutigen Türken ist dabei nur eine der möglichen Konnotationen dieser Trennlinie zwischen dem Eigenen und dem als Bedrohung empfundenen Anderen. Die historische Kontinuität und Anpassungsfähigkeit dieses Musters der Abgrenzung durch den Begriff des Türken lässt sich durch fortlaufende Reaktualisierungen des Feindbildes seit dem 16. Jahrhundert nachzeichnen. Bemerkenswert ist darüber hinaus, dass dieses Motiv bereits durch Strukturen besetzt war, die zeitlich vor dem Türken lagen, und Bilder der Sarazenen, Hagarener oder allgemeiner des Antichristen in Konfrontation mit dem historischen Türken fortgeführt und angepasst wurden.

Trotz punktuell anzutreffender positiver Besetzung des Türken ist deutlich geworden, dass die negativen Konnotationen dieses Deutungsträgers überwiegen und für die eigene Standortbestimmung als europäisch relevant sind. Denn

4 S. die Einleitung dieses Bandes, S. 13, Anm. 4. 
nur durch ein Aushandeln der Trennlinie zwischen dem Türken und Europa ist es möglich gewesen, Europa als Abendland, Christenheit oder schlicht als vermeintlich zivilisiert zu definieren. Wenn also von Europa gesprochen wird, bedeutet das implizit auch, dass bestimmte Vergangenheitsbezüge ausgeschlossen, andere dafür normativ eingefordert werden. Das wird anhand der EU-Beitrittsverhandlungen mit dem heutigen Staat Türkei anschaulich, in denen die kulturelle Abgrenzung fortdauert und die Anerkennung des Völkermords an den Armeniern zum Prüfstein europäischer Zugehörigkeit erhoben wird. ${ }^{5}$ Andernfalls werden, wie die 2001 von der französischen Nationalversammlung verabschiedete loi mémorielle oder ein Beschluss des Deutschen Bundestages zur türkischen "Aufarbeitung" dokumentieren, unter dem Schlagwort europäische Erinnerung die Grenzen gegenüber der Türkei neu begründet. $^{6}$

In Roland Scheels Fallstudie zu den Wikingern arbeiten Vorstellungen vom Eigenen und Anderen auf andere Weise an der Grenze dessen, was Europa genannt werden kann. Im Unterschied zum Türken werden die Wikinger nicht explizit als Anderer Europas dargestellt. Dennoch ist die Europavorstellung auch in den hier angeführten Deutungsgeschichten auf indirekte Weise präsent. Die Wikinger funktionieren als Antonym zu Nachbarkonzepten Europas: nämlich dem Christentum oder der lateinischen Kultur. So wurde im 12. Jahrhundert der Mord eines heidnischen nordischen Seekriegers an einem christlichen, altenglischen König zum Ursprungsmythos eines eigenständigen, christlichen Islands. In der Chronistik des mittelalterlichen Islands wurde der Wikinger als innerer Barbar aus dem eigenen Selbstbild verbannt, indem er einer fernen Vergangenheit zugewiesen wurde. Hier wurde Alterität in der Grundlage ihrer Entstehung eingeebnet, weil sie einen Selbstwiderspruch zu einem neuen Identitätskonzept bildete. Der Wikinger wurde in seiner Erinnerungsgeschichte zu einem domestizierten Anderen, der als solcher heute, von allen Assoziationen der Gewalt befreit, als neu erfundener harmloser Barbar auch in das Selbstbild Europas integriert werden kann.

Am Beispiel der isländischen Geschichtsschreibung zeigt diese Fallstudie ferner, wie die vormittelalterlichen nordischen Seekrieger eine negative narrative Rolle ausfüllten, die zum Beispiel in späteren, hochmittelalterlichen Er-

5 S. die folgenden Ausführungen zur Anerkennung von Völkermorden als konstitutives Element der Zugehörigkeit zu Europa.

6 S. z. B. Alain-Gérard Slama, La culpabilité de l'Occident. Les négociations d'adhésion avec la Turquie commenceront le 3 octobre 2005, in: Le Figaro, 20.12.2004; Carsten Fiedler, Union verlangt von der Türkei Mäßigung in Armenien-Debatte, in: Die Welt, 23.04.2005, und Heinrich August Winkler, Überdehntes Wir-Gefühl. Als Wertegemeinschaft kann die EU nur Nationen umfassen, die sich der politischen Kultur des Westens vorbehaltlos öffnen, ebd., 28.12.2005. 
zählungen mit muslimischen Piraten besetzt wurde. Vor dem Hintergrund der Fallstudie zum Türken wird deutlich, wie damit ein asymmetrischer Gegenbegriff zum Identifikationsbegriff Europa aufgerufen wurde, der im Sinne Reinhart Kosellecks primär auf Selbst- und nicht auf Fremdwahrnehmung beruhte und die implizit angesprochene Gegenseite für eigene Identitätskonzepte funktionalisierte. ${ }^{7}$ Der Wikinger stand für Eigenschaften, die gefürchtet sind und daher externalisiert wurden. Sie gehörten zwar im isländischen Fall der eigenen Vergangenheit an, verblieben jedoch in einer hinreichend fernen Vergangenheit, die für die jeweilige Gegenwart die binäre Logik des Feindbildes Wikinger aufhob. Im Erinnerungsmodus der Traditionsstiftung folgt aus dieser Historisierung, dass die Figur des Anderen eingehegt werden konnte - nicht jedoch als der Andere als solcher, sondern aus der Retrospektive eines scheinbar definitiv überwundenen Konflikts im Falle der Wikinger oder in der Erwartung einer erneuten Zuspitzung des dualistischen Feindbildes mit Blick auf den Türken.

Auch im Falle Versailles' haben sich die Erinnerungskonflikte nach dem Zweiten Weltkrieg scheinbar definitiv aufgehoben. Die von Friedemann Pestel untersuchten »Interpretationsübungen« der architektonisch-künstlerischen Schlossausstattung im Modus der Deutungsregie haben im deutsch-französischen Wechselspiel von Triumph und Niederlage allerdings ein solches Maß an Analogie zueinander entwickelt, dass eine qualitative Unterscheidung in der nach 1945 erfolgten wechselseitigen Anerkennung der nationalen Interpretationen kaum noch möglich ist, da sie nahezu vollkommen historisiert worden sind. Folglich markiert Versailles gegenwärtig keine räumliche Exklusionslinie zwischen Deutschland und Frankreich mehr, sondern stellt die Frage nach Inklusion und Exklusion nur noch außerhalb davon. Wenn sich Großbritannien und Irland beim Élysée-Jubiläum 2003 aus dem Europaverständnis des deutschfranzösischen Gespanns bewusst ausgeschlossen fühlen und sich stattdessen auf partikulare beziehungsweise transatlantische Rahmen beziehen, tritt an die Stelle der Tradition einer deutsch-französischen Feindschaft die europapolitisch aufgeladene Vorstellung einer aus der Konfliktgeschichte umgedeuteten gemeinsamen Hegemonie.

Bleibt aufgrund einer hohen semiotischen Verfügbarkeit der bislang betrachteten Deutungsträger die dichotome Logik von Feindbildern in ihrer Grundstruktur erhalten, wandelt sie sich, wenn angesichts der deutlich größeren zeitlichen Nähe ein höheres Konfliktpotenzial bewältigt werden muss. Im Modus des Deutungskampfs tritt in Gregor Feindts Fallstudie zu Flucht und Vertreibung

7 Reinhart Koselleck, Zur historisch-politischen Semantik asymmetrischer Gegenbegriffe, in: Ders., Vergangene Zukunft. Zur Semantik geschichtlicher Zeiten, Frankfurt am Main 1979, S. 211 -259. Vgl. weiterführend Kay Junge/Kirill Postoutenko (Hrsg.), Asymmetrical Concepts after Reinhart Koselleck. Historical Semantics and Beyond, New Brunswick 2011. 
daher innerhalb einer solchen Konfliktkonstellation die Figur des Anderen in gewandelter Form auf, indem sie als Dritte Instanz zur Überwindung der Dichotomie führt. Bevor dieses Erinnerungsmuster im vierten Schritt dieses Vergleichs hinsichtlich seines Umgangs mit Konflikthaftigkeit genauer analysiert wird, soll es hier mit Blick auf die ihm inhärenten räumlichen Vorstellungen betrachtet werden.

In den Europa-Vorstellungen öffentlicher Interventionen von Wissenschaftlern steht Europa im Zusammenhang mit Flucht und Vertreibung für eine vorweggenommene und anerkannte Instanz des Anderen. Hier ist zu erkennen, wie Wissenschaftler einen multiperspektivischen Zugang auch auf Erinnerungsfragen übertragen und durch Politikberatung letztlich Modelle von Erinnerung anleiten. ${ }^{8}$ Mit der aus der Wissenschaft entliehenen Forderung nach einer multiperspektivischen Erinnerung erhält der erinnerte Andere - repräsentiert durch Europa - eine aktive Funktion, wird folglich als erinnernder Dritter zu einer appellationsfähigen Akteursinstanz, die als Leitbild einer europäischen Erinnerung dient. Im Streitfall des geplanten "Zentrums gegen Vertreibungen" beziehen sich alle Akteure auf einen Erinnerungsraum Europa, um ihre spezifischen Deutungen zu legitimieren, und entleeren ihn dadurch semantisch. In dieser Einhegung von Erinnerungskonflikten stellt Europa folglich eine Alternative $\mathrm{zu}$ untereinander unvereinbaren - hier: nationalen - Erinnerungen dar, indem es durch die explizite Anerkennung anderer Erinnerungsinhalte selbst zu einem multiperspektivischen Dritten wird.

Aus diesen Abgrenzungen und Grenzziehungen sind in den unterschiedlichen Fallstudien Räume Europas zu erkennen, die als diskursive Realitäten Europa einen Inhalt geben. Die offensichtlichste Konkretisierung ist die Unterscheidung zwischen Osteuropa und Westeuropa, die keineswegs erst seit Bestehen des Eisernen Vorhangs die Europawahrnehmung strukturiert. ${ }^{9}$ Die dabei implizierte Unterscheidung von Zentren und Peripherien des Kontinents lässt sich an den mit europäischer Erinnerung bezeichneten Inhalten selbst erkennen. So zeigt Klaus Oschemas Fallstudie zu Karl dem Großen, wie das karolingische Frankenreich nach dem Zweiten Weltkrieg die westeuropäische Integration traditionsstiftend als europäisch unterfütterte. Durch die explizite Gleichset-

8 S. die Fallstudie von Gregor Feindt, S. 169.

9 Zur Abgrenzung eines instabilen »Osteuropas« von einem "westeuropäischen« Zivilisationsmodell im Zuge der Staatsgründungen nach dem Ersten Weltkrieg s. Peter Bugge, , Shatter Zones: The Creation and Re-creation of Europe's East in Ideas of Europe since 1914, in: Menno Spiering/Michael J. Wintle (Hrsg.), Ideas of Europe since 1914. The Legacy of the First World War, Basingstoke 2002, S. $47-68$, hier S. 47. Eine Verortung im Westen dominiert auch noch das gegenwärtige Europabild, s. Vanessa Conze, Das Europa der Deutschen. Ideen von Europa in Deutschland zwischen Reichstradition und Westorientierung (1920-1970), München 2005, S. 1. 
zung mit dem Herrschaftsgebiet Karls des Großen grenzte sich das Europa der Sechs vom Ostblock und Kommunismus ab, ganz dem Muster der asymmetrischen Gegenbegriffe folgend: Ein mit Karl legitimiertes historisch-westkirchlich-lateinisches Europa verstand sich dabei als eine »westeuropäische Schicksalsgemeinschaft ${ }^{10}{ }^{0}$ und nahm für sich in Anspruch, Europa als solches zu sein, bedurfte dazu aber der selbstvergewissernden Abgrenzung von einem »Anti«oder »Nichteuropa».

Diese dichotomische Vorstellung von Europa nutzt ebenso den aus der Erinnerung an den Türken geläufigen Bollwerk-Mythos. Die Konstruktion einer antemurale christianitatis beschreibt die Abwehr eines expansiven Feindes. Doch bildete im Kontext des Kalten Kriegs die Bedrohung nicht mehr der Islam, der durch langfristige Kulturkontakte und Migration teilweise zu einem domestizierten, auch im Inneren lebenden Anderen transformiert wurde. Stattdessen wurde die identitätskonstitutive Funktion des äußeren Anderen nunmehr politisch definiert; an die Stelle des Türken trat als neue Bedrohung die Sowjetunion. Diese Substitution des Türken durch den Bolschewisten hat auch, wie Marcin Napiórkowski zeigt, in der Erinnerung an den Warschauer Aufstand stattgefunden. Die Verteidigung der europäischen Zivilisation verläuft hier an der Weichsel gegen den Kommunismus in Gestalt der herannahenden Roten Armee. ${ }^{11}$

Der Transfer von Deutungsmustern wie dem Bollwerk transportiert die darin ausgedrückte räumliche Vorstellung. Im Abwehrkampf gegen die Türken war es zunächst nicht Europa, das es zu schützen galt, sondern die Christenheit gegen einen heidnischen und daher friedensunfähigen Erbfeind. Mit der Debatte über einen EU-Beitritt der Türkei in den 1990er-Jahren sind solche Vorstellungen zurückgekehrt, nun als christliches Erbe oder christlich-jüdische Wurzeln Europas formuliert. Sie verweisen auf eine dezidiert christliche Vorstellung des Abendlandes, die Europa nach 1945, mit Karl dem Großen als Gewährsmann, politisch auf die Erstunterzeichner der Römischen Verträge zentrierte. ${ }^{12}$

Spätestens mit der Zäsur von 1989 haben diese funktionalen Äquivalenzen

10 S. die Fallstudie von Klaus Oschema, S. 57.

11 Eine ähnliche Kopplung von Antibolschewismus und der Verteidigung der Kultur des Abendlandes stellt auch Axel Schildt, Zwischen Abendland und Amerika. Studien zur westdeutschen Ideenlandschaft der 50er Jahre, München 1999, S. 22, fest.

12 Zur historischen Semantik der Begriffe Abendland und Europa s. Heinz Hürten, »Europa und Abendland - Zwei unterschiedliche Begriffe politischer Orientierung«, in: Philipp W. Hildmann (Hrsg.), Vom christlichen Abendland zum christlichen Europa. Perspektiven eines religiös geprägten Europabegriffs für das 21. Jahrhundert, München 2009, S. 9-16, hier S. 10. Nichtsdestotrotz geht die Bedeutung des Abendlands nicht in seiner christlichen Komponente auf. Insbesondere in der jungen Bundesrepublik war der Begriff immer auch ein politisch-ideologisches Konzept der Westintegration Schildt, Zwischen Abendland und Amerika, S. 22 f., sowie Conze, Das Europa der Deutschen, S. 57. 
von religiösen und säkularen Europasemantiken mit Blick auf das kommunistische Feindbild ihre konstitutive Referenz verloren. Mit der symbolischen Überwindung des Systemgegensatzes ist die Kategorie Europa unter den Erwartungsdruck einer vermeintlichen Rückkehr des Ostens geraten. ${ }^{13}$ Die politischen Erweiterungen der Europäischen Union wirken sich, wie am Warschauer Aufstand deutlich wird, auch auf die Handlungsspielräume und Legitimationsressourcen von Erinnerung und Geschichtspolitik aus. So plädieren polnische Politiker und höchst unterschiedliche zivilgesellschaftliche Akteure seit Mitte der 2000er-Jahre vehement für eine in Europa, das heißt in der Europäischen Union, anerkannte und lebendige Erinnerung an den Warschauer Aufstand. Solche Forderungen sind nicht nur als Deutungskampf vor dem Hintergrund einer polnischen Repräsentation in einem vermeintlichen Kanon europäischer Erinnerung zu verstehen, sondern betreiben eine Deutung des Aufstandes selbst als europäisch.

Daniela Mehlers Fallstudie zu Srebrenica hingegen zeigt, wie das Anliegen eines gesamteuropäischen Gedenktags an das Massaker sowohl bei Bosniaken als auch seitens des Europäischen Parlaments auf Interesse gestoßen ist. Die Einreihung Srebrenicas in ein Narrativ negativer Erinnerung fällt leicht: Indem beide Seiten die Gewalteruption in der Region als Negation der europäischen Idee deuten, sprechen Bosniaken und Vertreter der Europäischen Union seit 1995 vom größten Kriegsverbrechen seit Ende des Zweiten Weltkriegs in Europa. Mit der Einrichtung des Gedenktags an den Völkermord hat das Europäische Parlament 2009 erinnerungspolitisch die Rückkehr des zerfallenen Jugoslawiens nach Europa vollzogen, symbolisch die politische Integration Bosnien-Herzegowinas in die Europäische Union vorweggenommen und das Versagen europäischer Konfliktregelung korrigiert.

Auch in der deutschen Debatte über das geplante »Zentrum gegen Vertreibungen « mobilisieren die Opferverbände die Zielvorstellung Europa, um ihrem Anliegen Legitimität zu verleihen. So verbindet die vom "Zentrum gegen Vertreibungen" initiierte Ausstellung "Erzwungene Wege» die Erinnerung an deutsche Heimatvertriebene mit der Erinnerung an andere als europäisch verstandene Zwangsmigrationen im 20. Jahrhundert. Deutsche Opfer werden so neben Armenier, Griechen und Türken, Juden oder Jugoslawen gestellt, die ebenso unter Gewaltandrohung ihre Heimat verließen, sodass sich Flucht und Vertreibung in die Gewalterfahrungen des 20. Jahrhunderts einreiht. Diese parallelisierende Europäisierung hat jedoch die Deutung der deutschen Erfahrung von Flucht und Vertreibung in keiner Form beeinflusst, sondern lediglich geholfen, deren im Kern aus den 1940er- und 1950er-Jahren stammende Interpretation der Zwangsmigration in ein neues Licht zu stellen.

$13 \mathrm{Zu}$ den Herausforderungen dieser Rückkehr s. Bugge, ,Shatter Zones`, S. 61 - 64 . 
Räumliche Europavorstellungen greifen auch auf entgrenzte Raumkategorien zurück, in denen europazentrierte Deutungsmuster aufgehen. Zwar gibt es auch hier Zentren und Peripherien, allerdings ist Europa in Bezug auf universalisierende Ansprüche nur eine Möglichkeit der räumlichen Verortung. Wenn Karl der Große einem Chronisten zu Beginn des zehnten Jahrhunderts als pater orbis galt und Versailles in seinem erdteilübergreifenden Bildprogramm die gloire universelle Ludwigs XIV. repräsentierte, wurde explizit über den europäischen Kontinent hinaus Deutungsmacht beansprucht. Während im Falle Karls dessen christliche Herrschaft als idealtypisch verstanden wurde, galt Ludwigs Repräsentation anderen Souveränen als wegweisend. Der Raumbezug liegt in beiden Fällen aber nicht im Globalen im Sinne der Anerkennung einer anderen epistemischen Struktur als der eigenen. Universal wird nicht in einer territorialen Dimension gedacht, sondern vielmehr aus Europa abgeleitet zum Idealtyp erhoben.

Solche universalen Deutungsprojektionen blieben allerdings einseitig: Weder bezogen außereuropäische Akteure Stellung zu dieser Vereinnahmung, noch waren deren Reaktionen überhaupt beabsichtigt. Folglich fehlte es Universalisierungen in ihrer außereuropäischen Dimension an Konflikthaftigkeit, oder sie wurden in späteren Deutungen explizit europäisiert: »Welt«kulturerbe wurde Versailles dank seiner »europäischen « Ausstrahlung. Anders als die gewöhnlich als Äquivalenzen verstandenen Begriffe Europa und Abendland beruhen diese universalen Erinnerungsmuster auf einem asymmetrischen Verhältnis zwischen der Kernkategorie Europa und einer als größer imaginierten räumlichen Einheit, auf die die darin artikulierten Deutungsansprüche konzentrisch ausgreifen. Um spätere bis hin zu gegenwärtigen Europavorstellungen zu fundieren, müssen die jeweiligen Deutungskategorien zeitlich-semantisch übersetzt werden. Gegenüber der Gefahr, dass sie dadurch für spätere Akteure unverständlich und daher irrelevant werden, dominiert aber häufiger ihre vermeintliche Suggestivkraft, die ihnen eine vorweggenommene Bestätigung ihres eigenen europäischen Erwartungshorizonts zu bieten scheint.

Was sich in diesen Fällen also beobachten lässt, sind Entgrenzungen räumlicher Kategorien, die entlang der Außengrenzen dieser Räume stattfinden. Sie beschränken sich jedoch nicht auf kulturelle Hegemonie. Vielmehr können sich Entgrenzungen regelrecht imperial weiten und konkrete territoriale Ansprüche kommunizieren. Im Falle von Flucht und Vertreibung gaben die vorherrschende Interpretation des völkerrechtlichen status quo und die politischen Sagbarkeitsregeln vor, ob dem »deutschen Osten « über seine Bedeutung als »kulturelles Erbe« auch eine jeweils gegenwärtige Relevanz als verlorenes Territorium zukam. Im Feindbild des Türken kehren sich dagegen die Raumrelationen teilweise um. Hier wird ein Abendland in Abwehrhaltung gegen vermeintlich universale Expansionspläne eines Gegners imaginiert, der verdächtigt werden 
kann, mit inneren Feinden im Bunde zu stehen - im 17. Jahrhundert waren diese aus Habsburger oder Reichssicht Spanien oder Frankreich, bei Anders Behring Breivik sind es »Multikulturalisten« oder »Globalisten«.

Die trotz der unterschiedlichen politischen Kontexte unveränderte Implikation dieser als universal wahrgenommenen Bedrohung besteht in der Forderung nach innerer Disziplinierung eines Europas jenseits konfessioneller oder dynastischer Polarisierungen oder jenseits eines als zersetzend gedeuteten Pluralismus. Die Entgrenzungen überwinden somit innere Bruchlinien der Europasemantiken und heben - gerade in Bedrohungsszenarien - die Zentrum-Peripherie-Relation zeitweilig auf.

\section{Kapital Europa}

Neben den impliziten Raumvorstellungen fungieren Deutungen als europäisch in einer zweiten Dimension als Zugang zu einem breiteren Deutungskampf. In diesen Zuschreibungen wird Europa zu einem symbolischen Kapital in heutigen Erinnerungsdebatten. Durch den Europabezug wird es möglich, partikularen Erinnerungen in einem größeren Kontext Relevanz zu verschaffen, daraus Forderungen abzuleiten oder sie außerhalb des eigenen Rahmens überhaupt kommunizierbar zu machen.

Wenn die Bosniaken 1993 den Bosnienkrieg und ab 1995 dezidiert Srebrenica als erneuten Völkermord in Europa bezeichneten, appellierten sie mit der Referenz an die Verantwortung europäischer Regierungen und internationaler Institutionen für den weiteren Konfliktverlauf. Sie setzten auf die normative Kraft Europas, in dem nach Ende des Zweiten Weltkriegs und dem Holocaust Völkermord keinen Raum mehr haben dürfe. Wie soeben anhand der Zäsur 1989 angedeutet, traf die Nachfrage der Bosniaken nach einer Zugehörigkeit zu Europa auf ein Bedürfnis bei den angerufenen Akteuren, nicht nur in der langfristigen Perspektive der »Rückkehr des Ostens« nach Europa, sondern zur Lösung konkreter kurz- und mittelfristiger Problemlagen von unmittelbarer politischer Relevanz für die EU-Mitgliedsstaaten. Denn eine Beitrittsperspektive für die Länder des westlichen Balkans verhieß nach Srebrenica als Fanal des Scheiterns internationaler Befriedungsversuche eine nachhaltige Lösung der Flüchtlingsproblematik und eine Stabilisierung der EU-Außengrenzen. Als Schritte auf diesem Weg rehabilitierte die EU mit dem Abschluss des Stabilisierungsabkommens, einer Beitrittspartnerschaft und der Einsetzung des EUSonderbeauftragten für Bosnien-Herzegowina auch ihre Außenpolitik. Auf symbolischer Ebene wurde Srebrenica mit der Einrichtung eines europaweiten Gedenktags zur identitätsstiftenden Ressource einer negativen europäischen Erinnerung. 
Auch polnische Medien erhoffen sich von einer europäischen Deutung des Warschauer Aufstandes dessen Wahrnehmung in einer europäischen Erinnerung an den Zweiten Weltkrieg, die aus ihrer Perspektive mit klaren Freund-FeindSchemata einhergeht. Dies ist unter anderem als eine Reaktion darauf zu verstehen, dass weiten Teilen der Bevölkerung außerhalb Polens zwar der Warschauer Ghettoaufstand des Jahres 1943 bekannt ist, nicht jedoch die Erhebung des Spätsommers 1944. Indem der Warschauer Aufstand also nicht als europäisch wahrgenommen wird, kommt ihm eine vermeintlich zweitrangige Rolle zu. Während am Beispiel Srebrenica die Mobilisierung des Europabezugs einen positiven Effekt sowohl für die Bosniaken als auch für die Europäische Union hat, liegt der Mehrwert der Einschreibung in ein europäisches Narrativ im polnischen Fall lediglich auf nationaler Ebene vor, da die Einlösung der kommunizierten Ansprüche vonseiten der EU-Institutionen oder einer europäischen Öffentlichkeit noch aussteht.

Über symbolisches Kapital hinaus können sich Deutungen als europäisch auch konkret monetär lohnen, wenn dadurch finanzielle Mittel aus Förderprogrammen der Europäischen Union beansprucht werden können, die zum Teil den eigenen politischen Erwartungshorizont vorwegnehmen, also bereits vor einem formellen Beitritt zur politischen Union Zugehörigkeit attestieren. Die 1993 eröffnete »Viking route« wird beispielsweise als »europäischer Kulturweg» bereits seit 1987 gefördert - Jahre bevor Finnland und Schweden, durch die die Route verläuft, 1995 der EU beitraten. Andere erinnerungspolitische Akteure nehmen das Label Europa aufgrund seines normativen, vor allem aber finanziellen Mehrwerts auch bewusst in Anspruch: Die Institutionalisierung des "Europäischen Netzwerks Erinnerung und Solidarität« oder die Pläne eines "Zentrums gegen Vertreibungen« wären ohne die diskursive Inanspruchnahme Europas nicht nur über den nationalen Rahmen hinaus, sondern auch finanziell undenkbar gewesen.

Durch die Auslobung von Fördertöpfen schaffen die Europäischen Institutionen gezielt Anreize für europäische Selbstzuschreibungen, die sich auf Antrag offizialisieren lassen. So vergibt die Stadt Aachen im Vorfeld der Verleihung des Hauptpreises einen Jugendkarlspreis, bei dem der Frankenkaiser als erinnerungspolitischer Bannerträger fungiert, »um Jugendliche in der EU zur aktiven Mitgestaltung Europas anzuregen ${ }^{14}$. Vergeben wird er von der Stadt Aachen in Zusammenarbeit mit dem Europäischen Parlament, das auf diese Weise die europäische Selbstintegrationspraxis von Jugendlichen fördert. Durch solche Selbstzuschreibungen als europäisch gelingt es den Akteuren, im Erfolgsfall Anerkennung bei einer breiter als europäisch legitimierten Gemeinschaft zu finden. Bedingung dafür ist, dass die beiden symbolischen, aber eben auch 
monetären Währungssysteme zueinander äquivalent sind. Daraus folgt aber auch, dass in diesem Prozess des permanenten Aushandelns neue Grenzen gesetzt werden, wodurch, mutatis mutandis, anderen Akteuren und damit Deutungsträgern das Europäische zwangsläufig versagt bleibt. Jede Inklusion markiert zugleich eine Exklusion.

\section{Der ideologische Horizont Europas}

Der Appell an Europa funktioniert in Erinnerungsdiskursen immer wieder als Scharnier zu politischen Weltanschauungen. Akteure, die ihre Vergangenheitsdeutungen als europäisch präsentieren, positionieren sich damit auch gegenüber den weltanschaulichen Grenzlinien, welche die unterschiedlichen nationalen Öffentlichkeiten durchziehen. Bereits Mitte der 1980er-Jahre trat Europa auch in den spätsozialistischen Ländern des Ostblocks als Orientierungsgröße und Erwartungshorizont auf den Plan. Nicht nur in der oppositionellen Debatte um Mitteleuropa, sondern auch in staatskonformen Diskursen diente Europa als bedeutungsoffene Chiffre für Wandel, Fortschritt und eine Zukunft über Staats- und Blockgrenzen hinaus. ${ }^{15}$ Sind diese Staaten nach dem Zusammenbruch des Staatssozialismus im eigenen Verständnis sinnbildlich »nach Europa zurückgekehrt ${ }^{16}$ und unterfüttern ihre Transformation auch mit der Appellation an Europa, markiert ihr Europaverständnis gleichzeitig eine Position gegenüber einer wiedererlangten nationalen Option.

In diesem Kontext wird in den serbischen und polnischen Debatten um Srebrenica und den Warschauer Aufstand in gegenwärtigen Selbst- und Fremdzuschreibungen Europa an liberale Positionen gekoppelt und von konservativen Positionen abgegrenzt. Das ideologische Oppositionspaar liberal/ konservativ findet sein geschichtspolitisches Pendant im Gegensatz von Europa und der Nation als jeweils präferierten Rahmen von Erinnerung. In Polen, wo 2004 der EU-Beitritt mit dem 60. Jahrestag des Warschauer Aufstandes zusammenfiel, steht Europa für nationalkonservative Politiker als Chiffre für ein progressiv-liberales Szenario, in dem die Erinnerung an die leidvolle und

15 Christian Domnitz, Europäische Vorstellungswelten im Ostblock. Eine Topologie von Europanarrationen im Staatssozialismus, in: José M. Faraldo/Paulina Gulińska-Jurgiel/Ders. (Hrsg.), Europa im Ostblock. Vorstellungen und Diskurse (1945-1991), Köln 2008, S. 61 82, hier S. 76-80.

16 Die Formel geht auf den tschechoslowakischen Dissidenten und späteren Staatspräsidenten Václav Havel zurück. Václav Havel, Rede im polnischen Sejm und Senat, in: Ders., Angst vor der Freiheit. Reden des Staatspräsidenten, Reinbek bei Hamburg 1991, S. 19-29, hier S. 25. 
identitätsstiftende Vergangenheit Polens einem bloßen Versprechen des Fortschrittes geopfert werde und die Nation dahinter zurücktrete. ${ }^{17}$

Am serbischen Beispiel wird erkennbar, wie Europa bei Liberal- und Sozialdemokraten die eigene geschichtspolitische Position zu Srebrenica unterstreichen soll. Die von liberaler Seite geforderte Anerkennung der eigenen Schuld erscheint zugleich als Türöffner zu Europa und ist damit als Weg zur Erfüllung des typischerweise liberal konnotierten Werts des Fortschritts zu verstehen. Analog dazu disqualifizierte die serbische Regierung unter Boris Tadić die "patriotisch « gesinnten Oppositionsparteien, die sich vehement gegen die Verabschiedung einer Resolution zur Verurteilung des Massakers von Srebrenica aussprachen, als antieuropäisch und somit als rückständig.

Ein Perspektivwechsel auf die historische Dimension der europäischen Integration und die Geschichte der Europäischen Union kennzeichnet diese ideologische Dichotomie jedoch als osteuropäische Binnensicht. Außerhalb Osteuropas funktioniert Europa in geschichtspolitischen Diskursen keineswegs immer als eindeutiges ideologisches Kriterium, um liberale von konservativen Positionen zu unterscheiden. Europa ist also weder eine argumentative Waffe, die vorschnell einem vermeintlich liberalen geschichtspolitisch-rhetorischen Arsenal zugeordnet werden kann, noch funktioniert Europa pauschal innerhalb konservativer Diskurse als negativ besetzter Gegensatz zur positiv besetzten Nation. In geschichtspolitischen Debatten ist Europa vielmehr ein Mittel, mit dem gerade konservative Kräfte ihre Positionen der Brandmarkung als nationalistisch und rückständig entziehen und in einer Weise artikulieren können, die als politisch korrekt empfunden wird.

So hat in Deutschland der Bund der Vertriebenen seinem Narrativ von Flucht und Vertreibung in dem Moment eine Zukunft sichern können, in dem das Schicksal deutscher Opfer ein - und in der deutschen Wahrnehmung das einzig konkretisierte - Beispiel eines europäischen "Jahrhunderts der Vertreibungen" wurde. Auf ähnliche Weise ist es den Organisatoren des Katholikentags in Wien 1983 gelungen, mit dem Verweis auf die gemeinsame christliche Vergangenheit und Zukunft Europas einer befürchteten Parallelisierung mit dem Jubiläum von 1933 und dessen nationalistischer Vereinnahmung zu entgehen. ${ }^{18}$ Indem Europa nationale Denkmuster überwindet, führt es eine eigene Logik in das öffentliche Sprechen von der Vergangenheit und das Aushandeln von Erinnerung ein,

17 Diese Dichotomie von Europa als Westeuropa mit liberalen Werten und einem konservativen und autokratischen Osteuropa existiert in der Fremdzuschreibung seit der Zwischenkriegszeit, s. Bugge, `Shatter Zones`, S. 51. Die Gleichsetzung Europas mit »freiheitlichpluralistischen Werten" wird ähnlich als Resultat eines längeren historischen Konfliktes betont, in dem ehemals konkurrierende Europavorstellungen vereinnahmt wurden. Conze, Das Europa der Deutschen, S. 4.

18 S. die Fallstudie von Simon Hadler, S. 97. 
welche die unterschiedlichen Akteure der Erinnerung unabhängig von ihrer ideologischen Ausrichtung für sich nutzen.

\section{Europas Heterogenitätsbewältigung}

Der Diskurs über europäische Erinnerung ist, das haben wir in der Einleitung gezeigt, auch ein Diskurs über den Umgang mit Andersartigkeit und dadurch bedingte Konflikte. Insbesondere die Fallstudien zu Erinnerung als Deutungskampf thematisieren Vergangenheitsdeutungen, die in zeitlicher Nähe zu gewaltsamen Geschehnissen stehen. Ihre Zusammenschau zeigt für das 20. Jahrhundert eine Wandlung der Erinnerungen an gewaltsame Konflikte. Europa wird zum semantischen Kondensat dieser Wandlung.

Standen sich in der Deutung vergangener Konflikte gewöhnlich dessen teilnehmende Parteien nicht nur als erinnerte, sondern auch als erinnernde Antagonisten gegenüber und schrieben so den betreffenden Konflikt fort, hegt diese gewandelte Form der Konflikterinnerung die ursprüngliche Auseinandersetzung ein. Diese Veränderung lässt sich als Zusammenspiel und gegenseitige Transformation von drei unterschiedlichen Deutungsmustern nachzeichnen: Erstens zeigt sich, wie der Dualismus von Triumph und Niederlage, der die dichotomische Struktur des Konflikts erinnernd perpetuiert, in Erinnerungsprozessen zunehmend an Bedeutung verliert. Zweitens verändert sich das Erinnerungsmuster von Täter- und Opferschaft, sodass die dichotome Struktur des Konflikts zeitweilig nivelliert wird, Opfer und Täter also für sich stehen können. Schließlich lässt sich beobachten, wie auf dieser Grundlage durch die Vorstellung einer Dritten Instanz ein neues Muster unparteilichen Erinnerns erschlossen wird, das die dichotome Struktur des Konflikts grundsätzlich zu überwinden versucht. Diese Einhegung von Konflikten stellt zugleich den Erwartungshorizont dar, den der Begriff Europa heute in Erinnerungsdiskursen eröffnet.

Triumph und Niederlage sind die beiden Pole eines Deutungsmusters, das für die Repräsentation historischer Konflikte gerade im Paradigma nationaler Erinnerung lange vorherrschte. Die Fallstudie zu Versailles illustriert dies besonders deutlich. Die erinnernden Inszenierungen deutsch-französischer Konflikte arbeiteten bis zur deutschen Besatzung im Zweiten Weltkrieg mit einer scharfen Kontrastierung von Triumph und Niederlage, die den vergangenen Konflikt in seiner Erinnerung fortschrieb und für zeitgenössische Konflikte nutzbar machte. So schlugen sich die militärischen Triumphe Ludwigs XIV. im 17. Jahrhundert in der symbolischen Ausgestaltung des Versailler Schlosses nieder und wurden in der Glorifizierung der Siege des revolutionären Frankreichs im Nationalmuseum des 19. Jahrhunderts in der Erinnerung vergegen- 
wärtigt. Während der Pariser Vorortkonferenzen 1919 beeinflusste das Dekor unmittelbar die Inszenierung der Unterzeichnung des Friedensvertrages mit dem Deutschen Reich. Darüber hinaus konnte die Zurschaustellung militärischen Erfolgs auch neu zu begründende politische Einheiten untermauern, wie sich in Anton von Werners Darstellung der Kaiserproklamation nachvollziehen lässt.

Zugleich erfuhr Triumph als Deutungsmuster mit dem Versailler Vertrag eine erste Zäsur, betonte dessen Kriegsschuldparagraf doch in zuvor unbekannter Weise das Leid des Kriegs gegenüber seinem Ruhm. Damit begann eine Wandlung der Kriegserinnerung von einer heroischen Konflikterinnerung hin zu einer Erinnerung an Gewalt, und damit verbunden an die Opfer des Krieges. Die binäre Logik des Konflikts wurde so um eine passivere ${ }^{19}$ Wahrnehmung von Opferschaft ergänzt, wobei Triumph, Niederlage und Leid zunächst nebeneinander standen. ${ }^{20}$ Der Zweite Weltkrieg und die Massenvernichtung in den Lagern der Nationalsozialisten verstärkten diese Verschiebung grundlegender Deutungsmuster von militärischen Konflikten weiter, spitzten die Fokussierung auf Gewalt zu und führten zu einem völlig neuen Verständnis von Massengewalt und Völkermord. ${ }^{21}$ Statt wie bisher die Kriegserfahrung als Bestandteil einer glanzvollen Siegesgeschichte zu erinnern, trat in der deutenden Verarbeitung vor dem Hintergrund eines zuvor undenkbaren Ausmaßes an zivilen Opfern, infrastruktureller Zerstörung und allen voran des Holocaust die Gewalterfahrung ins Zentrum von Erinnerung. ${ }^{22}$ So wurde der Opferstatus, der nach 1945 den Juden zunehmend unbestritten zuerkannt wurde, in der erinnernden Aneignung vergangener Gewalterfahrung zu einem erstrebenswerten Prädikat. Dass dieser Status dabei auch die eigene Täterschaft zu nivellieren vermochte, zeigt das

19 Karsten Fischer, Between Sacrification and Victimization. On Political Semantics and Its Strategic Functions, in: Uwe Ewald/Ksenija Turkovic (Hrsg.), Large-Scale Victimization as a Potential Source of Terrorist Activities. Importance of Regaining Security in Post-Conflict Societies, Amsterdam 2006, S. 67 - 72, hier S. 69.

20 Für den höheren Stellenwert des Opfergedenkens infolge des Ersten Weltkriegs s. Jay Winter, Sites of Memory, Sites of Mourning. The Great War in European Cultural History, Cambridge 1995.

21 Der vom polnisch-jüdischen Juristen Rafał Lemkin 1943 definierte Begriff des >Völkermords`, der in der Konvention über die Verhütung und Bestrafung des Völkermordes der Vereinten Nationen Aufnahme fand, kann als Beispiel hierfür dienen. Lemkin entwickelte den Begriff vor dem ursprünglichen Hintergrund des Völkermords an den Armeniern ab 1915 und konkretisierte ihn aus der Erfahrung des Zweiten Weltkriegs. Vgl. Christian Werkmeister, Johannes Lepsius und die Verbrechen an den Armeniern. Die Vorgeschichte der UN-Genozidkonvention, in: Sybille Steinbacher (Hrsg.), Holocaust und Völkermord. Die Reichweite des Vergleichs, Frankfurt am Main/New York 2012, S. 83-104.

22 Jan-Werner Müller, Contesting Democracy. Political Thought in Twentieth-Century Europe, New Haven 2011, S. 126, und Peter Reichel, Auschwitz, in: Étienne François/Hagen Schulze (Hrsg.), Deutsche Erinnerungsorte I, München 2002, S. 600-621. 
Beispiel der frühen Bundesrepublik, in der nicht nur Vertriebene, sondern die Deutschen in ihrer Gesamtheit als Opfer des Nationalsozialismus gedeutet wurden. ${ }^{23}$

Diese Entwicklungen manifestieren sich in der Gegenwart europäischer Erinnerung, in der die Möglichkeiten einer triumphalen Konflikterinnerung stark eingeschränkt sind und nur im Falle eines vermeintlichen Gegensatzes zu Europa möglich bleiben, wie die triumphale Rhetorik gegenüber überwundenen totalitären Diktaturen zeigt. Darüber hinaus bleibt der triumphale Gestus als Ausdrucksform im Repertoire europäischer Erinnerung erhalten, wenn er sich auf die ferne Vergangenheit bezieht. Im Modus der traditionsstiftenden Erinnerung, so hatten wir in der Einleitung argumentiert, besteht bis in unsere Gegenwart hinein ein größerer Möglichkeitsraum unterschiedlicher Interpretationen, und insofern kann in diesem Modus die zurückgedrängte Triumpherinnerung eine beschränkte Präsenz wahren. Dies wird deutlich, wenn der Oberbürgermeister von Graz oder der Bischof von St. Pölten den Sieg der europäischen Zivilisation gegen eine türkische Bedrohung beschwören. ${ }^{24}$

Parallel zur zunehmenden Marginalisierung der Triumpherinnerung im Kontext der beiden Weltkriege ist anhand der Fallstudien auch eine Veränderung in der Erinnerung an Opfer zu beobachten. Der Triumpherinnerung entsprach eine Erinnerung an Opfer im Sinne eines sacrificium, also der Ehrung der eigenen Gefallenen als Helden, die sich für Volk und Vaterland aktiv geopfert hatten, um damit einem höheren Zweck zu dienen. ${ }^{25}$ Die ungarische Erinnerung an das Ende der Belagerung von Kőszeg durch die Türken illustrierte bei der 400-Jahr-Feier 1932 mit dem Verweis auf den »Schandfrieden« von Trianon die Erfolglosigkeit und damit letztlich Unmöglichkeit eines solchen Narrativs. Auch im polnischen Stalinismus war die Erinnerung an das Heldentum des Warschauer Aufstandes letztlich nur in systemkritischen Kreisen möglich, denen die Kommunisten vorwarfen, mit der Erhebung die polnische Hauptstadt aus politischen Motiven der Zerstörung preisgegeben zu haben.

Mit der Umdeutung vergangener Konflikte, die ohnmächtiges Erleiden und irrationale Gewalt in den Vordergrund stellte, trat an die Stelle des sacrificium das Deutungsmuster der victima, also des passiven und wehrlosen Opfers. Dies veranschaulicht die Erinnerung an Flucht und Vertreibung, bei der sich Betroffene mit der Formel des Heimatverlustes als unschuldige Opfer deuteten. Ein solches Verständnis der Heimatvertriebenen als victimae schloss zugleich eine

23 Reinhart Koselleck, Die Diskontinuität der Erinnerung, in: Deutsche Zeitschrift für Philosophie 47 (1999), S. 213 -222, hier S. 215.

24 Vgl. die Fallstudie von Simon Hadler, S. 96.

25 Vgl. Herfried Münkler/Karsten Fischer, "Nothing to kill or to die for...». Überlegungen zu einer politischen Theorie des Opfers, in: Leviathan 28 (2000), H. 3, S. 343-362, hier S. 345. 
Betrachtung der nationalsozialistischen Besatzungspolitik in Osteuropa oder ethnischer Konflikte vor 1939 aus und fokussierte sich allein auf deutsche Opfer.

Zeitgleich und mit wiederholter Wechselwirkung wandelte sich durch die Erinnerung an den Zweiten Weltkrieg und den Holocaust das Deutungsmuster der victima zu einer Erinnerung, die nunmehr das Leid anderer mit einschloss. Sie produzierte neue normgebende und erfolgversprechende Formen der Erinnerung, die sich auch in anderen Fallstudien nachverfolgen lassen. ${ }^{26}$ Gerade die Klage Bosnien-Herzegowinas vor dem Internationalen Gerichtshof im Jahr 1993 zeigt, dass sich mit Bezug auf den Holocaust ein universalisierter Opferdiskurs ${ }^{27}$ und ein Modell "globaler Opferschaft ${ }^{28}{ }^{2}$ institutionalisieren ließen, die über internationale Organisationen und Nichtregierungsorganisationen Verbreitung gefunden haben. Die bosnische Regierung versuchte mit expliziten Verweisen auf den Holocaust und den Zweiten Weltkrieg, einen Analogieschluss zwischen den muslimischen Bosniern und den weltweit erinnerten jüdischen Opfern herzustellen, um so die eigenen Kriegstoten als unschuldige Opfer zu deuten und die Kriegshandlungen gegen Bosnien-Herzegowina als Völkermord zu qualifizieren. In der deutschen Debatte um den Bundeswehreinsatz im Kosovokrieg erfuhr der Opferdiskurs schließlich sogar eine präventive Ausweitung. Mit dem Verweis auf die Gefahr eines zweiten Auschwitz genügte in der Umkehrung der bisherigen Logik bereits die drohende Erfahrung von Massengewalt als Interventionskriterium.

Dabei bedeutet der Einschluss des Anderen als ebenso Leidendem, dass mit Referenz auf Europa weder ein Heldengedenken noch das Erinnern an ein sacrificium möglich ist. Der begrenzte Wirkungsgrad solcher konstitutiver Regeln zeigt sich allerdings gerade in den vermeintlichen Grenzregionen europäischer Erinnerung. Bosniakisch-nationalen Politikern ist es im staatlichen Erinnern gelungen, den international als passives Opfer gedeuteten Tod von 8.000 muslimischen Männern in Srebrenica zugleich für den eigenen sozialen Raum in ein heldenhaftes, religiös unterlegtes Selbstopfer umzudeuten. Diese unterschiedlichen Deutungen im Inneren und im Äußeren widersprechen sich grundlegend und können nicht zusammengeführt werden. Doch sind sie in der Fremd- und

26 Vgl. Daniel Levy/Natan Sznaider, Memories of Europe: Cosmopolitanism and Its Others, in: Chris Rumford (Hrsg.), Cosmopolitanism and Europe, Liverpool 2007, S. 158-177, hier S. 163 f., mit Blick auf die Verarbeitung von Unrechtserfahrungen im Kolonialismus s. Michael Rothberg, Multidirectional Memory. Remembering the Holocaust in the Age of Decolonization, Stanford 2009.

27 Münkler/Fischer, »Nothing to kill«; Daniel Levy/Natan Sznaider, Erinnerung im globalen Zeitalter. Der Holocaust, Frankfurt am Main 2001, S. 155- 158.

28 Thorsten Bonacker, Globale Opferschaft. Zum Charisma des Opfers in Transitional Justice Prozessen, in: Zeitschrift für Internationale Beziehungen 19 (2012), H. 1, S. 5-36. 
Eigenwahrnehmung für die bosniakische Nation gleichermaßen konstitutiv und veranschaulichen in ihrer Unvereinbarkeit die Grenze europäischer Erinnerung.

Die Denkfigur der passiven victima nivelliert durch die inhärente Individualisierung der Gewalterfahrung nicht nur die Dichotomie des Konfliktes, sondern auch die Bedeutung der historischen Konfliktparteien für eine gegenwärtige Erinnerung. Finden wir also in allen der hier versammelten Fallstudien im Modus des Deutungskampfs eine zentrale Bedeutung der victima, bezieht diese oftmals die Anerkennung anderer erinnerter Opferschaften mit ein. Aus der Unmöglichkeit des Triumphes und der Universalisierung des Opfers folgt so die Bereitschaft und die Erwartung eines Dialogs zwischen den unterschiedlichen europäischen Erinnerungsgruppen, der an der Wende zum 21. Jahrhundert Europa mit Assoziationen von Unparteilichkeit und Zivilisierung aufgeladen hat. Ein solches Europa überschreitet also jegliche erinnerte Konfliktsituation und überwindet sie durch die alleinige Hervorhebung unschuldiger Opfer. Als Argument in Erinnerungsdiskursen wandelte sich Europa im Laufe des 20. Jahrhunderts von einer dezidierten Parteinahme für einen partikularen kulturellen Typus, wie zum Beispiel das christliche Abendland als Gegenposition zum Kommunismus im Europa der Sechs, zu einer über den Konflikt erhabenen Position der Unparteilichkeit. Diese Universalisierung Europas verläuft nicht zufällig in zeitlicher wie räumlicher Hinsicht parallel zur voranschreitenden Integration europäischer Staaten, sondern ist deren identitätskonkretes und in die Vergangenheit projiziertes Äquivalent.

So ist diese Entwicklung auch im Zusammenhang mit dem generationellen Übergang von der Erlebnisgeneration des Zweiten Weltkriegs zu einer Generation von Nachkriegsintellektuellen zu sehen, die typischerweise mit der Teilung des europäischen Kontinents durch den Kalten Krieg sozialisiert worden ist. Daraus erklärt sich, dass Europa beispielsweise in der Debatte um das "Zentrum gegen Vertreibungen" mehr als nur ein Argument ist und für Wissenschaftler und Intellektuelle als unparteiliche Instanz zum Erwartungshorizont einer dialogischen Erinnerung wird. ${ }^{29}$ Innerhalb des virulenten Erinnerungskonflikts kann diese Beanspruchung wissenschaftlicher Sachlichkeit damit die Autorität eines entperspektivierten Standorts einnehmen. Die europäische Stimme im Erinnerungskonflikt erschließt so die Position eines Dritten, der keiner der vorhandenen Konfliktparteien zuzuschreiben ist und mit ver-

29 Ein Beispiel für diesen Anspruch bietet - auch ohne konkreten Bezug auf Europa - Hans Henning Hahn, Geschichtspolitik und binationale Beziehungen. Plädoyer für erinnerungspolitischen Verhaltenskodex, in: Ders./Robert Traba (Hrsg.), Deutsch-polnische Erinnerungsorte 4, Paderborn 2013, S. 159-173. Zur generationellen Verhaftung des Forschungsfeldes Erinnerungsorte s. Gregor Feindt/Félix Krawatzek/Daniela Mehler/Friedemann Pestel/Rieke Trimçev, Entangled Memory. Toward a Third Wave in Memory Studies, in: History and Theory 53 (2014), S. $24-44$, hier S. 39. 
meintlich gleichem Beobachtungsabstand auf sie blickt. Genau dieses Erinnerungsmuster verspricht, die dichotome Struktur des Erinnerungskonfliktes nicht nur im Modus der Viktimisierung zeitweilig zu nivellieren, sondern den Konflikt tatsächlich einzuhegen. ${ }^{30}$

Ähnlich wie die politisch engagierte Wissenschaft im Streit um Flucht und Vertreibung wird Europa auch im Erinnerungskonflikt zu Srebrenica mit der Vorstellung eines unparteilichen Dritten verbunden. Jedoch wurde es nicht erst an einem bestimmten, generationell bedingten Punkt einer von Konflikten geprägten Erinnerungsgeschichte zu einer nichtbetroffenen Instanz, sondern diese Qualität war der Erinnerung an Srebrenica bereits von ihrem Beginn an inhärent. So versuchte die europäische Diplomatie ab 1991, in den Jugoslawienkriegen für die internationale Gemeinschaft zu vermitteln. Später etablierten aus den europäischen NATO-Staaten stammende Blauhelmsoldaten Schutzzonen in Bosnien-Herzegowina. Auch die juristische Bewältigung der Kriege ging auf eine Initiative zurück, in deren Institutionalisierung europäischen Staaten und Institutionen die Rolle eines vermeintlich unparteilichen Dritten zufiel.

\section{Europa als erinnerungspolitischer Akteur}

In der Einleitung ist bereits deutlich geworden, dass die Bedeutung von Europa als Argument in Erinnerungsdiskursen auch von der Position abhängt, welche die Europasemantik in einer Vergangenheitsdeutung einnimmt. Die traditionsstiftende Pragmatik europäischer Erinnerungen an Karl den Großen und den Türken, oder, um typische Beispiele aus dem breiteren öffentlichen Diskurs anzuführen, die Antike, die Renaissance oder die Aufklärung, setzt den Europabegriff als Attribut solcher Deutungsträger ein. ${ }^{31}$ Darüber hinaus, das wurde bereits anhand der Figur des Dritten deutlich, kann der Europabegriff in Erinnerungshandlungen solche Handlungen selbst als europäisch qualifizieren.

Europa kann jedoch nicht nur Attribut des Deutungsträgers oder einer Erinnerungshandlung sein, sondern auch Attribut des erinnernden Akteurs. Nicht

30 In dieser Transformation von Erinnerungsmustern spiegeln sich damit auch eine im Laufe des 20. Jahrhunderts zunehmende Prominenz der Figur des Dritten und der Übergang von dichotomen zu triadischen Denkformen besonders in der Soziologie und Sozialtheorie wider. Dazu Thomas Bedorf/Joachim Fischer/Gesa Lindemann (Hrsg.), Theorien des Dritten. Innovationen in Soziologie und Sozialphilosophie, München 2010, und Eva Eßlinger/ Tobias Schlechtriemen/Doris Schweitzer/Alexander Zons (Hrsg.), Die Figur des Dritten. Ein kulturwissenschaftliches Paradigma, Berlin 2010.

31 Symptomatisch dafür Thomas Ferenczi, L’Europe, sa mémoire, ses valeurs, in: Le Monde, 18.02.2004; Marcel Detienne, Un lungo filo sembra unire i Greci e noi [Ein langer Faden scheint die Griechen und uns zu verbinden], in: La Repubblica, 27.10.2007, und Quatorze voix fortes pour porter l'espérance, in: La Croix, 23.12.2011. 
nur im Falle der Europäischen Union oder des Europarates, sondern ebenso im Beispiel des "Europäischen Netzwerks Erinnerung und Solidarität« oder der Stadt Aachen wird Europa zum Teil der Selbstbeschreibung. ${ }^{32}$ Anhand dieser Beispiele lässt sich fragen, welche Regelveränderungen solche Zuschreibungen für die bereits analysierten europäischen Erinnerungen mit sich bringen.

Aus dem Selbstverständnis, genuin europäisch zu sein, richtete der Europarat mit dem Programm für europäische Kulturrouten eine »Viking Cultural Route« ein und hoffte so, nicht nur den regionalen Tourismus zu fördern, sondern zugleich das Bewusstsein für das europäische Erbe. Mit der Stiftung des Karlspreises inszeniert sich die Stadt Aachen, über die geografische Lage am Dreiländereck von Deutschland, Belgien und den Niederlanden hinaus, als »Wiege Europas« und als »Stadt der europäischen Einigung«. Auf diese Weise schaffen europäische Akteure nicht nur Orte, sondern verräumlichen Europa. Dabei verstehen politische Akteure bis hin zur Europäischen Union den Mehrwert des symbolischen Kapitals Europas insbesondere in seiner Auswirkung auf die kollektive Identität der Europäer. ${ }^{33}$ Das Bemühen um den "Schutz und die Entwicklung des kulturellen Erbes Europas « ${ }^{34}$ macht sie folglich $\mathrm{zu} »$ Agenten eines europäischen Bewusstseins « ${ }^{35}$.

Während dabei sowohl der Europarat, die Europäische Union als auch die Stadt Aachen eine eher kulturelle Deutung der Vergangenheit präsentieren, lässt sich an den aufgezeigten Ambivalenzen des unparteilichen Dritten auch illustrieren, wie sehr Europa als Akteur Erinnerung politisch beeinflussen kann. Dabei besteht Europa zunächst aus denjenigen Akteuren, die sich in einem situativen Zusammenschluss vereinigen. Das »Europäische Netzwerk Erinnerung und Solidarität " vermag es nicht, alle von Flucht und Vertreibung direkt betroffenen Staaten zu erfassen, und findet in seiner inhaltlichen Arbeit lediglich die Förderung einschlägiger Projekte als gemeinsamen Nenner. Die Selbstbeschreibung des Netzwerks, und stärker noch die verschiedenen, sich als europäisch verstehenden Zusammenschlüsse von Intellektuellen und Wissenschaftlern, ermöglichen einen Beitrag zur Debatte um Flucht und Vertreibung als unparteiliche Dritte.

Das Europäische wird hier als angemessenste Form des Dialogs zwischen

32 So bildet die Stadt Aachen auf ihrem Internetauftritt zur Selbstbeschreibung das Karlsdenkmal mit daneben wehender Europaflagge ab. Vgl. http://www.aachen.de (30.09.2013).

33 Camille Mazé, Des usages politiques du musée à l'échelle européenne. Contribution à l'analyse de l'européanisation de la mémoire comme catégorie d'action publique, in: Politique européenne 37 (2012), H. 2, S. $72-100$.

34 Vertrag von Lissabon Art. 3, $\$ 3$, http://europa.eu/lisbon_treaty/full_text/index_de.htm (30.09.2013).

35 Cris Shore, Building Europe. The Cultural Politics of European Integration, London/New York 2000, S. 26. 
Nationen präsentiert, die nationale Positionen zwangsläufig überlagert. Gerade diese Überschreitung und gleichzeitige Einengung national gerahmter Vergangenheitsdeutungen kann aber auch als konkrete Bedrohung wahrgenommen werden. In der Erinnerung an den Warschauer Aufstand folgt daraus in rechtskonservativen Kreisen die radikale Ablehnung Europas und seiner dialogischen Erinnerung. In der Erinnerung an Srebrenica handelt es sich beim unparteilichen Dritten um politische und internationale Akteure, die Europa auch unabhängig von den Jugoslawienkriegen für sich in Anspruch nehmen. Das manifestiert sich konkret, wenn die Europäische Kommission gegenüber den jugoslawischen Nachfolgestaaten eine Zusammenarbeit mit dem JugoslawienTribunal als Bedingung für eine Annäherung an die Europäische Union formuliert. Auch die Einrichtung des europaweiten Gedenktags kann als disziplinierendes Instrument gegenüber Serbien gewertet werden. Europäisches Erinnern wird somit zu einem impliziten Beitrittskriterium.

\section{Die Aporie unparteilicher Erinnerung als Gründungsfigur für Europa}

»Nie wieder!« - das ist das Leitmotiv des genuin europäischen Umgangs mit Konflikten und Unterschieden nach 1945, das schrittweise die Erinnerung auch auf globaler Ebene strukturiert. Nach den Grausamkeiten des Zweiten Weltkriegs wurde es politisch unbestreitbar, dass sich staatlich ausgeübter Terror, ethnische oder rassistisch motivierte Gewalt in Europa nicht wieder ereignen durften. Durch Wiederholung und Inszenierung im öffentlichen Raum entwickelte sich das Deutungsmuster des »Nie wieder!« zu einem normativen Erinnerungsparadigma, das sich einerseits schrittweise von seinem ursprünglichen Kontext löste ${ }^{36}$ und andererseits zu dem strukturierenden Merkmal europäischer Erinnerung avancierte.

Diese Regel des »Nie wieder!« hat ihre Durchsetzungskraft auch in der Erinnerungsforschung bewiesen. Die Einleitung dieses Bandes zeigte auf, wie sich die Debatte um europäische Erinnerung immer mehr zur Streitfrage um einen »negativen Gründungsmythos« verdichtet. Die vergleichende Betrachtung der

36 Den Vorgang der Entkontextualisierung des Holocaust hat Helmut Dubiel zutreffend unterstrichen: "Concepts, symbols, and images are taken out of their immediate context and are employed to code, in a single term, the collective pain that people inflict upon others." Dubiel, The Remembrance of the Holocaust as a Catalyst for a Transnational Ethic?, in: New German Critique 90 (2003), S. 59-70, hier S. 61. S. ebenfalls Klas-Göran Karlsson, The Holocaust in European Historical Culture, in: Teresa Pinheiro/Beata Cieszyńska/José Eduardo Franco (Hrsg.), Ideas of $\mid$ for Europe. An Interdisciplinary Approach to European Identity, Frankfurt am Main 2012, S. 427 -440, hier S. 433. 
Fallstudien unterstreicht, dass hierbei zwei konstitutive Elemente zu unterscheiden sind: Erstens geht Europa als symbolische Objektivierung in Erinnerungshandlungen regelhaft mit solchen Erinnerungsmustern einher, in denen vergangenen Konflikt- und Gewalterfahrungen von einem Standpunkt der Unparteilichkeit aus begegnet wird. Zweitens scheint dieses Versprechen auch insofern erfüllbar, als es im politischen System, im Rechts- und im Wissenschaftssystem vermehrt Akteure gibt, die mit Europa im Namen und im Namen Europas sprechen und so durch Institutionalisierung dem Standpunkt der Unparteilichkeit ein besonderes Gewicht verleihen. Europa symbolisiert also neben der Unparteilichkeitserwartung eine Instanz mit Handlungsmacht - die Erwartung wird somit zum Unparteilichkeitsversprechen.

Es war der Anspruch dieses Bandes, mithilfe der Heuristiken der Vielschichtigkeit und Vielstimmigkeit ${ }^{37}$ auch solche europäischen Erinnerungen sichtbar zu machen, die nicht im Mittelpunkt der ubiquitären Europäisierung von Erinnerungshandlungen stehen. Denn durch dieses Vorgehen, so haben die Fallstudien gezeigt, kann der sich abzeichnende Konsens zu europäischer Erinnerung kritisch auf seine blinden Flecken und Widersprüche hin befragt werden. Die Fallstudien zur Erinnerung an Srebrenica und den Warschauer Aufstand betonen mit Blick auf den Holocaust als integrierenden »negativen Gründungsmythos" und Kern einer europäischen Erinnerungskultur, dass es vielversprechend und notwendig ist, danach zu fragen, was europäische Erinnerung jenseits der gewohnten Argumentationsfiguren längst schon zu bedeuten begonnen hat.

Die Fallstudie zur Erinnerung an den Warschauer Aufstand umreißt das Feld eines Erinnerungskonfliktes, der ohne »dialogisches Echo« innerhalb des Diskurses über die europäische Weltkriegserinnerung der alten EU der 15 geführt wird. ${ }^{38}$ Auch wenn die Europareferenz für den Erinnerungskonflikt relevant ist, insbesondere um Erwartungen zukünftiger politischer Teilhabe und gesellschaftlicher Identität zu formulieren, ist die Europasemantik hier nicht deckungsgleich mit dem Imperativ des »Nie wieder!«, der europäische Erinnerung auszeichnet. Ganz im Gegenteil: Das Zentrum der Erinnerung an den Warschauer Aufstand bilden nationale Helden, deren sacrificium stark affirmativ gedacht wird. ${ }^{39}$ Zudem steht Europa auf doppelte Weise für das Vergessen: Aus

37 Vgl. die Einleitung dieses Bandes, S. 29-32.

38 Dieser »rideau de fer mémoriel « erstreckt sich Emmanuel Droit zufolge auch auf das »trou de mémoire« des Gulag im Westen. Droit, Le Goulag contre la Shoah: Mémoires officielles et cultures mémorielles dans l'Europe élargie, in: Vingtième Siècle 94 (2007), S. 101-120, hier S. $103-108$.

39 Dies erklärt die besondere Problematik für die Integration des Warschauer Aufstandes in eine europäische Meistererzählung. Denn nicht nur liegt er jenseits des »rideau de fer 
der Binnenperspektive des polnischen Diskurses funktioniert es als Marker gegen diejenigen geschichtspolitischen Positionen, die bereit sind, für die Anbindung an Westeuropa den Preis des Vergessens eigener Gewalterfahrungen zu zahlen. ${ }^{40}$ Gleichzeitig finden aber solche Stimmen, die sich positiv auf Europa beziehen, nach außen öffentlich keine Anerkennung. Zwar wird versucht, die eigene Erfahrung als europäisch zu präsentieren, jedoch gelingt es den Akteuren nicht, diese Position in den westlichen EU-Mitgliedsstaaten $\mathrm{zu}$ verankern. Vielmehr ist aus polnischer Perspektive ein "Übermaß an Taktlosigkeiten ${ }^{41}$ festzustellen, wenn die Siegermächte des Zweiten Weltkriegs wichtige Jahrestage des Aufstandes missachten und der Warschauer Aufstand in der westeuropäischen Öffentlichkeit nicht erscheint oder bestenfalls als Leerstelle Erwähnung findet. ${ }^{42}$ Trotz aller Bekenntnisse zur Einbindung Osteuropas in eine »europäische Erinnerungsgemeinschaft" seitens der Verfechter des Holocaust als "negativem Gründungsmythos ${ }^{43}$ kann die derzeitige Schlussfolgerung nur lauten, dass sich ein national als so zentral empfundener Erinnerungskonflikt wie der um den Warschauer Aufstand durch das von der Holocaust-Erinnerung geprägte Regelverständnis nicht in den Diskurs um europäische Erinnerung einfügen lässt.

Eine andere Herausforderung stellt die Erinnerung an Srebrenica dar. In ihr werden über die Vielschichtigkeit von Erinnerungsmustern die Grenzen des Narrativs europäischer Erinnerung, das sich auf den »negativen Gründungsmythos« des Holocaust beruft, deutlich. Dieser Fall steht exemplarisch dafür, dass bereits die ersten Erinnerungen an eine Erfahrung in sich selbst vielschichtig sind, indem sie auf frühere Deutungsträger und andere Erinnerungsmuster verweisen. ${ }^{44}$ Denn die Erinnerungsmuster des Deutungsträgers

mémoriel«, sondern durch das schwierige Heldengedenken passt er nicht in das Narrativ von Shoah und Gulag, die eine gemeinsame ethische Grundlage teilen. Ebd., S. $118 \mathrm{f}$.

40 S. auch die in der Einleitung dieses Bandes skizzierte Debatte um den Diktaturenvergleich nach der EU-Erweiterung von 2004, S. 23-25.

41 Zachodnia prasa o 60. rocznicy Powstania Warszawskiego [Die westliche Presse über den 60. Jahrestag des Warschauer Aufstandes], in: Gazeta Wyborcza, 02.08.2004.

42 So stellt der Aufstand nur eine Randerscheinung des Feuilletons dar oder wird - wie 1994 vom deutschen Bundespräsidenten Roman Herzog oder 2004 vom US-Verteidigungsminister Colin Powell - mit dem Aufstand im Warschauer Ghetto 1943 verwechselt. Vgl. Joseph Croitoru, Erbarmungslos pünktlich, in: Frankfurter Allgemeine Zeitung, 06.11.2004, und Timothy Snyder, A Rough Guide to an Uncertain Future, in: The Guardian, 19.11.2011.

43 Claus Leggewie, Der Kampf um die europäische Erinnerung. Ein Schlachtfeld wird besichtigt, München 2011, S. 12 und Aleida Assmann, Auf dem Weg zu einer europäischen Erinnerungskultur, Wien 2012, S. 45. Weniger emphatisch denn analytisch weist auch Tony Judt dem Holocaust die Funktion einer "Eintrittskarte « nach Europa zu, und im Gespann mit ihm dem Genozid an den Armeniern und Srebrenica. Judt, Postwar. A History of Europe since 1945, New York 2005, S. 803 f.

44 S. dazu in der Einleitung dieses Bandes, S. 32. 
Holocaust prägten bereits die unmittelbare Wahrnehmung des gewaltsamen Konfliktes. Die Kategorisierung als Genozid, welche als Objektivierung der Holocaust-Erinnerung im Medium des Rechts zu begreifen ist, stellt dabei eine Institution des »Nie wieder!« dar. Durch eine Anbindung an diesen Erinnerungsimperativ kann eine Aufarbeitung von Gewalterfahrungen analog zu der als erfolgreich empfundenen Erinnerung an den Holocaust befördert werden. Genau diese über ein institutionalisiertes Medium der Unparteilichkeit vermittelte Analogie erweist sich aber im Falle Srebrenicas nicht als konflikteinhegend oder gar konfliktüberwindend, sondern konfliktverschärfend. Denn die Fortschreibung wird von den Konfliktparteien selbst strategisch eingesetzt. Gerade der internationalen Gemeinschaft erlaubt dieses Erinnerungsmuster, von der Position der Unparteilichkeit des Völkerrechts zu sprechen - und dadurch den eigenen Akteursstatus im Konflikt und das möglicherweise begangene Unrecht auszublenden. Schon bevor der Holocaust, gipfelnd in der Stockholmer Erklärung 2000, mit dem Ziel gegenseitiger Verständigung zum »negativen Gründungsmythos Europas" gekürt wurde, war das ihm zugrunde liegende »Nie wieder! $\lll$ in der Erinnerung an Srebrenica gescheitert. ${ }^{45}$

Die Fallstudien zum Warschauer Aufstand und zu Srebrenica helfen, ein Problem zu identifizieren, das man die >Aporie des unparteilichen Erinnerns، nennen kann. Der "Dritte« als neu erschlossenes Muster eines anerkennenden Erinnerns macht deutlich, dass diese Figur für einen Deutungsträger erst im Kontext breiterer Transformationen in der Erinnerung an Gewalterfahrungen im Zusammenspiel und der Ablösung unterschiedlicher generationeller Erinnerungsmuster erschlossen wird. Die heutige Verkörperung dieser Position unparteilichen Erinnerns durch Europa aber läuft Gefahr, genau diese konstitutive Vielschichtigkeit, die Gewordenheit dieses konfliktüberwindenden Erinnerungsmusters, auszublenden. Wenn das Paradigma der Erinnerung an den Holocaust als »negativen Gründungsmythos Europas« durch seine Generalisierung und Abstraktion diese Vielschichtigkeit nicht transparent zu halten vermag, dann droht es sich der eigenen Grundlagen zu berauben.

45 Zur Stockholmer Erklärung als "Gründungsmythos der Europäischen Union« s. Dubiel, Remembrance of the Holocaust, S. 68. Mit Blick auf das Genozid-Argument für die deutsche Kosovo-Intervention s. Eckart Conze, Der Holocaust wird zum negativen Gründungsmythos des Kontinents. Deutschland im Schnittpunkt europäischer Geschichtszonen, in: Die Politische Meinung 56 (2011), H. 494/495, S. 14 -18, hier S. 18. Jüngst dazu mit weiterer symbolischer Verkürzung auf Auschwitz als das Symbol des Holocaust auch Karlsson, Holocaust, S. 427. 


\section{Schlussbetrachtung}

Unsere Zusammenschau der sieben Fallstudien dieses Bandes hat gezeigt, dass die Bedeutung europäischer Erinnerung umstrittener ist, als der öffentliche und wissenschaftliche Diskurs vermuten lassen. Vergleicht man die Gebrauchsmuster dieses semantischen Paars, so erscheint europäische Erinnerung als Terrain eines lebhaften und akuten Deutungskonfliktes. Auf diesem Terrain haben wir vier tertia comparationis herausgestellt, die den Deutungsstreit strukturieren und die wir mit Raumvorstellungen, Kapital, Ideologien und Heterogenitätsbewältigung betitelt haben. Sie verstehen sich als Anstoß, die Debatte über europäische Erinnerung aus der Teleologie von Vergemeinschaftung durch Erinnerung zu führen.

So erhält die polyfone Europasemantik durch die ihr eingeschriebenen Raumvorstellungen eine erste Kontur. Erinnerungskonflikte um Europa polarisieren soziale Räume von Erinnerung. Aus den von ihnen gezogenen Grenzen werden auch über den unmittelbaren Konflikt hinaus mental maps von Zentren und Peripherien erkennbar. Ihr wirkungsvollstes Zuordnungskriterium ist die Forderung nach Teilhabe an Europa - trotz der damit verbundenen Widersprüche eines homogen gedachten Raumes. Der expansive Charakter des selbsterklärten historischen Zentrums des Europas der Sechs entspricht dem normativen Anspruch des europäischen Erinnerungsprojekts, das heißt dem imperativen »Nie wieder!« der Holocaust-Erinnerung.

Jedoch lässt sich die Europasemantik nicht auf einen solchen politisch-normativ aufgeladenen Gehalt reduzieren. Durch die fortschreitende Institutionalisierung unterschiedlicher europäischer Akteure und deren wachsende Alltagspräsenz eignet sie sich als vielfältig wandelbare und im Diskurs anschlussfähige Referenz. Dadurch funktioniert Europa als symbolisches und auch monetäres Kapital. Im Bemühen, partikulare Deutungen durchzusetzen oder wenigstens anschlussfähig zu machen, setzen Akteure die Möglichkeit einer Deutung als europäisch offensiv ein. So kommt europäischer Erinnerung als Argument eine doppelte Funktion auf supranationaler und auf nationaler oder regionaler Ebene zu. Nur noch teilweise markiert Europa dabei die Trennlinie zwischen politisch-ideologischen Standpunkten.

Weitere Kontur erhält die Europasemantik durch den eng an den Begriff Europa geknüpften Deutungswandel von Konflikten. In prominenter Weise tritt hier aus der Nivellierung des Dualismus von Triumph und Niederlage die Figur eines unparteilichen Dritten hervor, deren Suggestionskraft in der Hoffnung auf eine grundsätzliche Einhegung erinnerter und zu erinnernder Konflikte besteht: Europäische Erinnerung wird heute gleichbedeutend mit »unparteilicher Erinnerung«.

Es ist jedoch bezeichnend, dass gerade Europa bejahende Akteure im 
selbsterklärten westeuropäischen Zentrum europäische Erinnerung als einen Kanon unstrittiger, aus dem "negativen Gründungsmythos « abgeleiteter Regeln der Konfliktaustragung verstehen. Im Mittelpunkt dieser als konsensual antizipierten Position steht eine universalisierende Interpretation vergangener Konflikte als Gewalterfahrung und die Betonung unschuldiger Opfer. Diese Konsensbeschwörung erweist sich allerdings nur als eine Position innerhalb eines Spektrums unterschiedlich verlaufender Inklusions- und Exklusionslinien des imaginierten Raumes Europa. Durch die Fallstudien wurde deutlich, dass insbesondere osteuropäische Erinnerungsakteure, die erst seit dem Zusammenbruch des Staatssozialismus und der Osterweiterung der EU dezidiert an europäischen Debatten partizipieren, auf die prekäre Zugehörigkeit zum Sprachspiel europäische Erinnerung verweisen. Sie geben Aufschluss über das Ringen um Regeln europäischer Vergangenheitsdeutung. Die hier zusammengeführten Konfliktlinien erhellen die ganz unterschiedlichen Bedeutungen und Funktionen, welche die Europasemantik in erinnerungspolitischen Diskursen heute annehmen kann.

Das diesem Band zugrunde gelegte Verständnis von europäischer Erinnerung als einer semantischen Realität unserer Gegenwart lässt statt einer antizipierten Konfliktüberwindung den per se konfliktiven Charakter von Erinnerung hervortreten. Europa als Argument für einen bestimmten Erfahrungsraum oder Erwartungshorizont erweist sich im Lichte systematischer Perspektivwechsel zwischen den beteiligten Akteuren als kontingent. Diese reflektieren so die Aporie einer Konsens- und Homogenitätserwartung des Postulats des "Nie wieder!

Die jeweiligen Europasemantiken und ihre in Vergangenheit wie Gegenwart begrenzte Erklärungsreichweite hängen auch vom Verhältnis von Erfahrungsund Erinnerungsobjektivierung ab, also der in der Einleitung beleuchteten Frage nach dem Zusammenhang von deutender und zeichenhafter Aneignung erlebter Gegenwart und erinnerter Vergangenheit. Wird dieses Verhältnis mithilfe der Heuristiken der Vielstimmigkeit und Vielschichtigkeit analysiert, erweisen sich scheinbar kontinuierliche Europabegriffe bei Deutungsträgern von hoher interpretatorischer Verfügbarkeit wie Karl dem Großen, dem Türken oder den Wikingern als Traditionsstiftungen voller Übersetzungsprobleme. Vermeintliche Deutungsgenealogien der longue durée im Falle von Versailles verlieren in ihren europäischen Übersetzungen an Kohärenz, wenn sie sich in vergleichender Perspektive als Eigendynamik einer Deutungsregie darstellen. Wenn sich schließlich in den Deutungen der Gewalterfahrungen von Flucht und Vertreibung, des Warschauer Aufstandes und Srebrenicas die Akteure europäischer Erinnerung an ihrem Vermögen zur Bewältigung von Heterogenität messen und Maßstäbe für unparteiliche Erinnerung zu setzen suchen, erweist sich 
die Vorstellung von wechselseitiger Opferanerkennung als Trugschluss eines Deutungskampfes unvereinbarer Positionen.

$\mathrm{Ob}$ diese Erinnerungskonflikte in Zukunft für Europa integrierend oder desintegrierend wirken - sicher ist nur, dass jeglicher Wandel aus veränderten Regeln der Konfliktaustragung hervorgehen wird. Solche Regelveränderungen, das haben die Beispiele gezeigt, geschehen durch Deutungsanalogien, die sich in ihren Verflechtungen der Kontrolle und Kalkulation seitens der Akteure entziehen. Folglich sind diese Regelveränderungen nicht mit dem Zielhorizont der Integration oder der Homogenisierung von Erinnerungen zu denken. Die politische, vor allem aber die wissenschaftliche Herausforderung lautet gerade nicht, durch den Appell an historische Erfahrungen alle Europäer an einem selbstreferenziellen Erinnerungsregime teilhaben zu lassen. Erinnerung - als vielstimmiges und vielschichtiges Phänomen verstanden - heißt, die Heterogenität Europas jenseits eines historischen Kanons und jenseits von Erinnerungsimperativen zu erschließen. Was eine solche Beschreibung leisten kann, ist, die Regeln der Konfliktaustragung transparent zu machen. Nur wenn Wissenschaft sich dieser Aufgabe stellt, kann sie gesellschaftliche Wandlungsprozesse reflexiv begleiten. 
